ARTICLE

https://doi.org/10.1038/s41467-018-08252-0

\title{
Lignin-polysaccharide interactions in plant secondary cell walls revealed by solid-state NMR
}

\author{
Xue Kang (D) ${ }^{1}$, Alex Kirui (1) ${ }^{1}$, Malitha C. Dickwella Widanage (i) ${ }^{1}$, Frederic Mentink-Vigier (1) ${ }^{2}$, \\ Daniel J. Cosgrove ${ }^{3} \&$ Tuo Wang (ID ${ }^{1}$
}

Lignin is a complex aromatic biopolymer that strengthens and waterproofs plant secondary cell walls, enabling mechanical stability in trees and long-distance water transport in xylem. Lignin removal is a key step in paper production and biomass conversion to biofuels, motivating efforts to re-engineer lignin biosynthesis. However, the physical nature of lignin's interactions with wall polysaccharides is not well understood. Here we show that lignin selfaggregates to form highly hydrophobic and dynamically unique nanodomains, with extensive surface contacts to xylan. Solid-state NMR spectroscopy of intact maize stems, supported by dynamic nuclear polarization, reveals that lignin has abundant electrostatic interactions with the polar motifs of xylan. Lignin preferentially binds xylans with 3-fold or distorted 2-fold helical screw conformations, indicative of xylans not closely associated with cellulose. These findings advance our knowledge of the molecular-level organization of lignocellulosic biomass, providing the structural foundation for optimization of post-harvest processing for biofuels and biomaterials.

\footnotetext{
${ }^{1}$ Department of Chemistry, Louisiana State University, Baton Rouge, LA 70803, USA. ${ }^{2}$ National High Magnetic Field Laboratory, Tallahassee, FL 32310 , USA.

${ }^{3}$ Department of Biology, Pennsylvania State University, University Park, PA 16802, USA. These authors contributed equally: Xue Kang, Alex Kirui.

Correspondence and requests for materials should be addressed to T.W. (email: tuowang@lsu.edu)
} 
T he secondary cell wall comprises the majority of plant biomass and is a sophisticated composite of cellulose, hemicellulose (mainly xylan and glucomannan) and lignin ${ }^{1,2}$. This supramolecular network formed by complex carbohydrates and aromatic polymers provides the cell with sufficient mechanical strength and rigidity, but it also makes the lignocellulosic materials inherently recalcitrant to chemical and enzymatical treatments during biofuel production ${ }^{3}$. Decades of efforts have been devoted to genetically engineering the plants to improve the composition and structure of cell wall polymers, aiming at increased digestibility ${ }^{4-6}$. This method offers the potential for effectively generating sustainable bioenergy, however, a major hurdle here is our inadequate understanding of the cell wall architecture on the molecular level.

In secondary cell walls of woody materials, bundles of cellulose microfibrils, typically $10-20 \mathrm{~nm}$ across 7 , are proposed to be coated by a xylan-lignin complex and crosslinked by glucomannans ${ }^{8-11}$. However, due to the inherent, technical constraints of traditional analytical methods, detailed molecular information about secondary cell wall organization has remained scarce. Conventional methods either rely on sequential extractions followed by compositional analysis or require partial dissolution of the lignocellulosic materials using organic solvents and ionic liquids for solution-NMR characterization ${ }^{12-14}$. These procedures substantially perturb the physical state and molecular interactions of biomolecules, introducing considerable uncertainty to our understanding of the cell wall structure. This limitation has been partially alleviated by the recent solid-state NMR (ssNMR) work that characterized xylan polymorphism in native secondary cell walls of Arabidopsis, revealing a two-fold helical screw symmetry with a regular pattern of acetate or glucuronate substitutions in cellulose-bound xylan ${ }^{15-17}$. However, many structural aspects still await investigation and a key question, examined here, is how lignin and polysaccharides are packed in intact secondary cell walls.

The atomic resolution of ssNMR spectroscopy and the sensitivity enhancement provided by the dynamic nuclear polarization (DNP) technique have enabled us to clarify and substantiate our ambiguous view of lignocellulose structure with detailed molecular evidence. The ${ }^{13} \mathrm{C}$-labeled stems from three energy and agricultural crops (maize, rice, switchgrass) as well as the model plant Arabidopsis were investigated. Dominant interactions between xylan with non-flatten conformations and lignin units rich in methyl ethers are observed whereas direct lignin-cellulose interactions are less prominent. Because the degree of hydration and timescale of motions are distinct between the lignin and polysaccharides, we propose that lignin self-aggregates in distinctive nanodomains with extensive surface contacts to hemicelluloses. These results provide a substantial revision of our understanding of the supramolecular architecture of secondary plant cell walls, which can facilitate the development of crops with higher digestibility and improve the efficiency of biomass deconstruction and conversion to biofuels.

\section{Results}

Polysaccharides and lignin are structurally polymorphic. Uniformly ${ }^{13} \mathrm{C}$-labeled stems of maize, rice, switchgrass, and Arabidopsis were produced for ssNMR analysis by growing the plants in a closed growth chamber with continuous supply of ${ }^{13} \mathrm{CO}_{2}$. Isotopic enrichment provides adequate sensitivity for systematically measuring two-dimensional (2D) ${ }^{13} \mathrm{C}-{ }^{13} \mathrm{C}$ spectra, which provide atomic resolution for determining the composition, subnanometer packing, site-specific hydration, and ns- $\mu$ s motion of lignin and polysaccharides in the near-native state.

Figure 1 shows representative $2 \mathrm{D}{ }^{13} \mathrm{C}^{13} \mathrm{C}$ correlation INADEQUATE spectra and molecular structures of biomolecules in intact maize stems. These samples contained predominantly secondary cell walls, as xyloglucan (a primary wall component) was negligible (Fig. 1a). The major hemicellulose, xylan, is dominated by its 2 -fold, extended conformers $\left(\mathrm{Xn}^{2 f}\right)$, accounting for $\sim 70 \mathrm{~mol} \%$ as indicated by the peak volume (Fig. 1b). This is potentially due to the extensive interaction of xylan with cellulose microfibrils, which promotes the two-fold, flat conformation. The interactions with cellulose rigidify the two-fold xylan and further enhance its signal in the current spectrum that relies on the ${ }^{1} \mathrm{H}$ ${ }^{13} \mathrm{C}$ cross-polarization (CP) to preferentially detect rigid components. These two-fold xylans are structurally polymorphic (Fig. 1a) and only a subgroup is in close contact with lignin as shown later. The 2 - and three-fold xylan conformers are both heavily acetylated and mixed on the sub-nanometer scale because a moderate cross peak exists between the $\mathrm{C} 1$ of two-fold xylan and the $\mathrm{C} 4$ of the three-fold conformer (Xn1 $1^{2 f}-\mathrm{Xn} 4^{3 f}$ ) (Supplementary Fig. 1a).

The glucan chains in cellulose microfibrils of secondary cell walls are highly polymorphic in structure. Among the six major allomorphs, types a-d are the internal chains while types $g$ and $f$ originate from surface residues (Fig. 1a). With the high resolution attained here, NMR further reveals subtle differences in cellulose organization in cell walls from growing coleoptiles versus mature stems (Fig. 1c) where microfibrils aggregate extensively $y^{7,18}$. The increase in relative intensities of the deeply embedded allomorph (type-c) and the reduced number of surface chains (Fig. 1c and Supplementary Table 1) collectively indicate the restructuring of elementary microfibrils on the aggregation interface, likely enabled by cooperative activity of multiple cellulose synthase complexes during secondary wall formation ${ }^{19,20}$.

Maize lignin is mainly composed of $p$-hydroxyphenyl $(\mathrm{H})$, guaiacyl (G), syringyl (S) and ferulate (FA) residues (Fig. 1e-g). The $\mathrm{H}, \mathrm{G}$, and $\mathrm{S}$ lignin units differ in the number of methoxyl substitutions at carbon 3 and 5, which results in well-resolved signals for the ring carbons. These aromatics bear conformational heterogeneity since they show broad linewidth and peak multiplicity (Supplementary Fig. 1). The ${ }^{13} \mathrm{C}$ NMR chemical shifts are documented in Supplementary Table 2, and the well-resolved signals of aromatics and carbohydrates enable further determination of their intermolecular packing as detailed below.

Lignin binds xylan through electrostatic interactions. Both native and modified lignins have been extensively studied using solution NMR ${ }^{13,14,21,22}$, but native interactions with other wall components are lost in the ball-milled, fractioned, and dissolved samples. Using the intact maize stems, we have measured a longmixing $(1.0 \mathrm{~s}) 2 \mathrm{D}$ spectrum, which displays 74 intermolecular cross peaks that are absent in the 0.1-s short-range correlation spectrum (Fig. 2a). This experiment provides exquisite details on the spatial proximities (instead of covalent bonding) of cell wall polymers as each cross peak represents a unique sub-nanometer contact between two distinct atoms in adjacent molecules. These intermolecular interactions are well-resolved in the NMR spectra and can be classified into four major categories depending on the interaction site and the structural motifs. First, cross peaks may occur between two different lignin units, for instance, the H4-S3/5 cross peak at $(159,153 \mathrm{ppm})$. Second, the methyl ethers of lignin may be spatially proximal to xylan and cellulose. Notable examples include the OMe-s4 at $(57,84 \mathrm{ppm})$ and the OMe$\mathrm{Xn} 4^{3 f}$ at $(57,78 \mathrm{ppm})$ caused by the contacts between lignin methyl ethers and surface cellulose carbon 4 and three-fold xylan carbon 4. Third, the aromatic carbons of lignin may correlate with the ring carbons of carbohydrates, for example, the S3/5$\mathrm{Xn} 4^{3 f}$ cross peak at $(153,78 \mathrm{ppm})$. Fourth, the xylan acetyl group may correlate with lignin as reflected by the $\mathrm{Ac}^{\mathrm{Me}}$-FA9 cross peak at $(21,169 \mathrm{ppm})$ and the $\mathrm{Ac}^{\mathrm{Me}}{ }_{-} \mathrm{S} 3 / 5$ cross peak at $(21,153 \mathrm{ppm})$, 
a

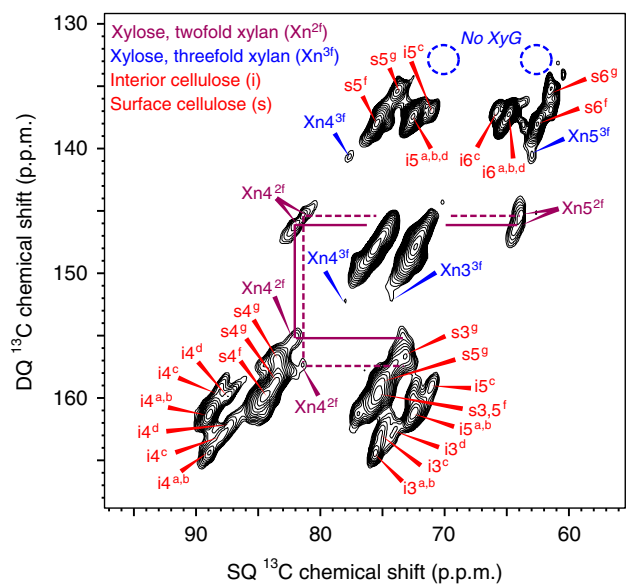

C

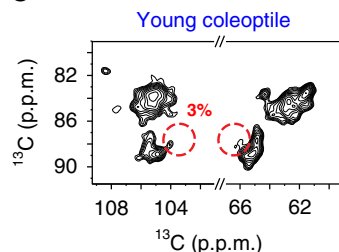

b

Twofold xylan $\left(\mathrm{Xn}^{2 f}\right)$

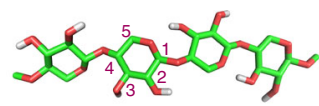

Threefold xylan $\left(\mathrm{Xn}^{3 f}\right)$



Cellulose

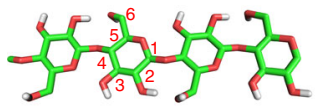

d

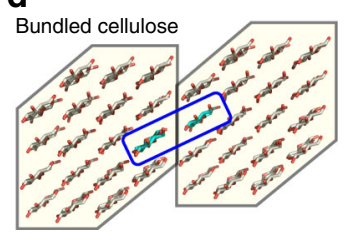

e

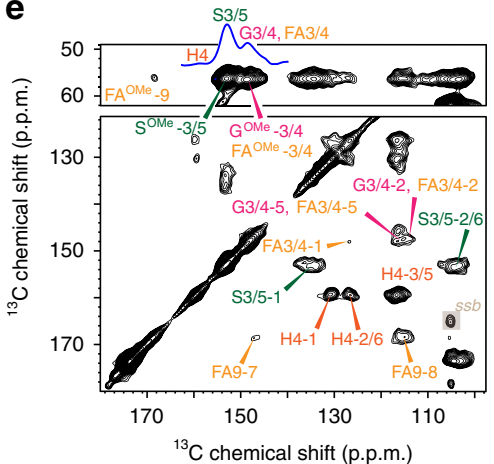

f

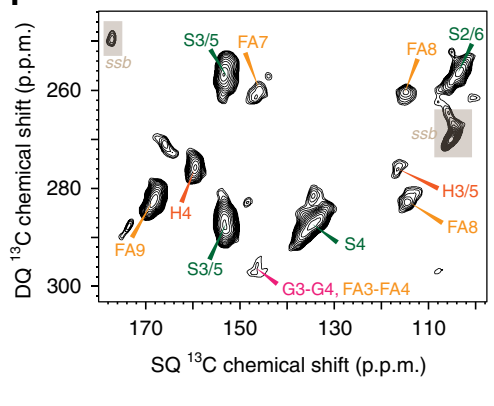

g<smiles>COc1ccccc1</smiles><smiles>COc1cccc(OC)c1</smiles>

Fig. 1 The polymorphic structure of lignin and polysaccharides in intact maize stems. a Representative $2 \mathrm{D}{ }^{13} \mathrm{C}-{ }^{13} \mathrm{C}$ correlation spectrum resolves the polymorphic signals of cellulose and xylan. The xyloglucan (XyG) signals are missing (blue circles), indicating a negligible amount of primary cell walls. Abbreviations are used for assignment, e.g., s4g is carbon 4 of glucose type-g on the microfibril surface. b Representative polysaccharide structures. c Cellulose signals in maize coleoptile and mature stems. The six cellulose allomorphs are labeled using letters $\mathbf{a}-\mathbf{g}$. The intensity of type-c cellulose is $2 \mathrm{X}$ greater in mature stems compared with coleoptiles. d An illustrative figure of two adjacent microfibrils, with the restructured chains boxed in blue, which increase the intensity of type-c cellulose in c. These chains restructure by changing the hydroxymethyl conformation. The number of glucan chains in the figure may not represent the actual microfibril structure. The aromatic signals of lignin are resolved using $2 \mathrm{D}{ }^{13} \mathrm{C}-{ }^{13} \mathrm{C}$ spectra measured using e $53-\mathrm{ms}$ CORD and $\mathbf{f}$ INADEQUATE methods. $\mathbf{g}$ Representative structures and ${ }^{13} \mathrm{C}$ chemical shifts of lignin

a

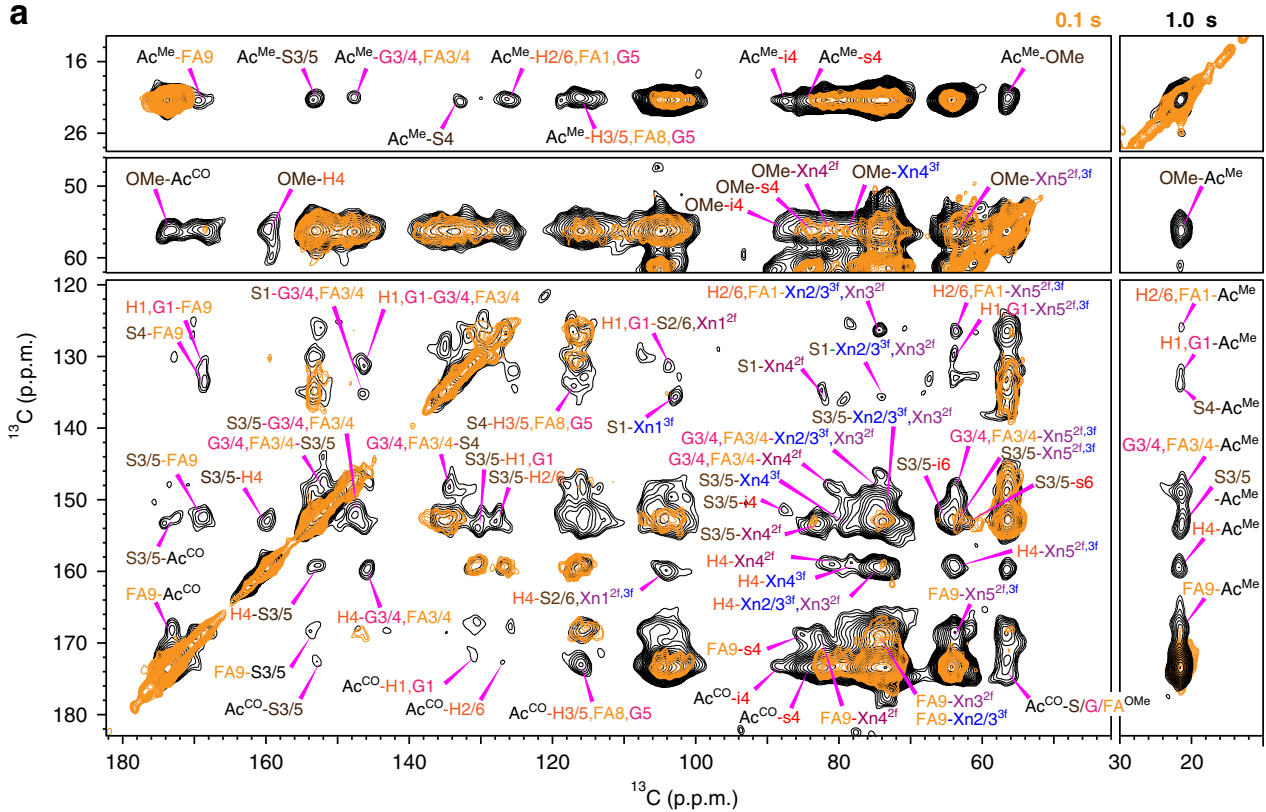

b

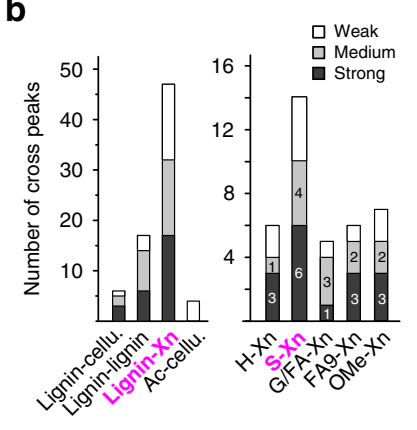

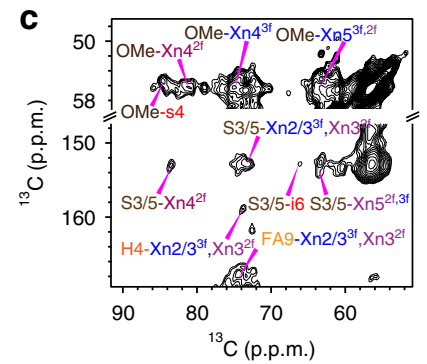

Fig. 2 Electrostatic interactions of xylan and S units dominate lignin-polysaccharide interactions in maize. a Representative ${ }^{13} \mathrm{C}-{ }^{13} \mathrm{C}$ spectra measured with short $(0.1 \mathrm{~s}$, orange) and long ( $1 \mathrm{~s}$, black) mixing times on maize. The 1-s spectrum shows many long-range intermolecular cross peaks that are absent in the 0.1 -s spectrum. Only intermolecular cross peaks are labeled. b Summary of the 74 intermolecular cross peaks in maize. For each category, the peaks are grouped by their strength. Cellulose is abbreviated as cellu. The interaction types with the most cross peaks are highlighted in magenta. Lignin-xylan contacts dominate, with the most cross peaks for S. Source Data are provided as a Source Data file. c The Xn-S and Xn-OMe cross peaks in the 100-ms spectrum reveal close contacts 
a

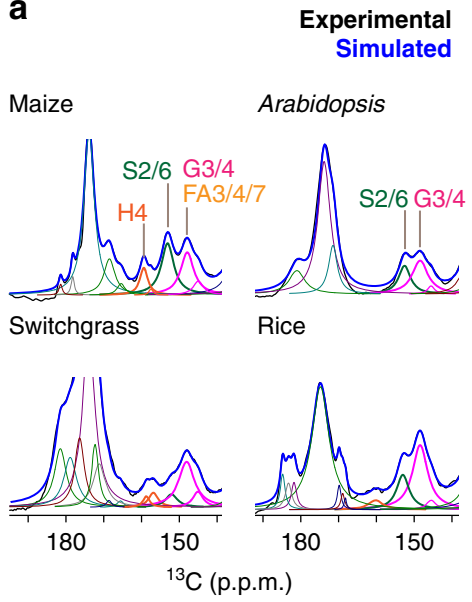

b



C

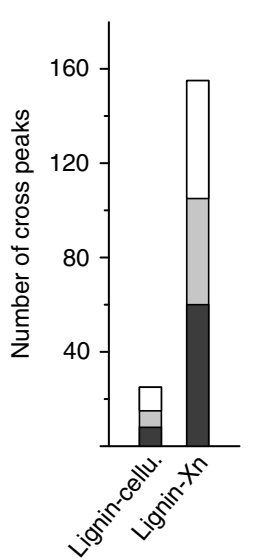

d

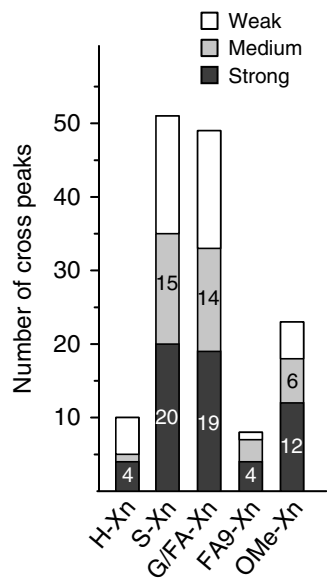





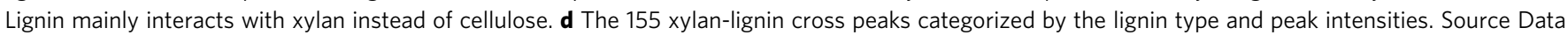
are provided as a Source Data file for Fig. 3b-d

which originate from physical contacts between the methyl carbon of xylan acetyl groups with the carbon 9 of ferulate and the carbon $3 / 5$ of syringyl units.

All intermolecular correlations are categorized by the interacted molecules and the relative intensities of these cross peaks (Fig. 2b). Xylan is the primary polysaccharide interactor of lignin with 47 cross peaks (Fig. 2b) and tightly contacts S-lignin $(<5 \AA)$, evidenced by cross peaks with only a short mixing period (Fig. 2c). Although cellulose-lignin contacts have been proposed in-silico ${ }^{23}$, the scarcity of cross peaks indicate few direct interactions in these non-woody samples (Fig. 2b).

We have extended these experiments to two other economically important grasses, switchgrass and rice, and to Arabidopsis, a model dicot (Fig. 3 and Supplementary Fig. 2). The lignin composition varies substantially among species: maize contains all the four units; in Arabidopsis $\mathrm{S}$ and $\mathrm{G}$ predominate; switchgrass lacks $\mathrm{S}$ and rice lacks $\mathrm{H}$ (Fig. 3a, b and Supplementary Fig. 1c). In these plants, we have identified 234 distinctive intermolecular cross peaks, and the large number of correlations identified here allows us to conduct statistical analysis of polymer contacts in this collection of plant species. Lignin has 155 cross peaks with xylan but only 25 contacts with cellulose, confirming the anchoring role of xylan (Fig. 3c). Despite its modest abundance (29\% on average) (Fig. 3b), the S-residue has the most abundant cross peaks with xylan (Fig. 3d). The amount of $\mathrm{G}$ and FA (52\%) is almost twice that of S but their xylan cross peaks are less prevalent. The number of physical contacts with polysaccharide correlates with the number of methyl ether groups $(\mathrm{OMe})$ in lignin and decreases in the order: $\mathrm{S}>\mathrm{G} / \mathrm{FA}>\mathrm{H}$. Consistently, $80 \%$ of the $\mathrm{OMe}-\mathrm{Xn}$ cross peaks are either strong or medium (Fig. 3d), indicating that electrostatic interactions between lignin methoxy groups and xylan polar functionalities dominate their physical contacts. The strong polysaccharide interaction of $\mathrm{S}$ units may be related to its early deposition during lignification $^{24}$ and the weak $\mathrm{H}-\mathrm{Xn}$ interaction enlightens the higher lignin extractability in H-rich plants 25.

Xylan with a non-flatten conformation binds lignin. The lignin-binding capacity of xylan is found to be conformationdependent. We measured a lignin-edited spectrum to selectively probe the interface between lignin and polysaccharide (Supplementary Fig. 3, 4). This challenging experiment is enabled by a 23-fold sensitivity enhancement (Fig. 4a) that is achieved using the DNP technique, which transfers polarization from electrons to NMR-active nuclei ${ }^{26-29}$. The lignin-bound polysaccharides contain xylan and part of the cellulose surface (Fig. $4 \mathrm{~b}$ ). In these maize walls, xylan in three-fold conformation constitutes less than one-third of all xylans but accounts for almost half of the lignin-bound xylans; therefore, three-fold conformation facilitates xylan-aromatic interactions. In contrast, the two-fold xylans are structurally polymorphic and lignin only binds subtypes b and c, whose signals are sandwiched between the flat conformer (type-a) and the three-fold allomorph. Since the xylan ${ }^{13} \mathrm{C} 4$ chemical shift indicates glycosidic bond conformation, it shows that the flatribbon structure of the two-fold conformation is not favorable for binding the intrinsically disordered lignin polymer.

Lignin self-aggregates to form a highly hydrophobic domain. To probe the hydration profile of secondary cell walls, we have compared the intensities of water-associated molecules with those of the whole cell wall (Supplementary Fig. 5). The relative intensities correlate with the extent of water retention and reveal a decreasing hydration gradient in the order: 3-fold xylan $>$ twofold xylan $\sim$ cellulose surface $>$ cellulose internal chains $>$ lignin (Fig. 5a). This experimental observation dovetails with a simulation reporting $50 \%$ faster diffusion for water molecules in lignin than those bound to xylan ${ }^{30}$. The distinctive hydration of lignin and three-fold xylan excludes homogeneous mixing, instead suggesting proximity of separate domains: lignin nanodomains close to a well-hydrated matrix of three-fold xylan (Fig. 6). The hydrated matrix further connects the junctions of two-fold xylan and surface cellulose ${ }^{31}$, two molecules with comparable hydration (Fig. 5a), thus bridging lignin with cellulose (Fig. 6).

Compared with primary cell walls ${ }^{32}$, secondary walls have considerably weaker water associations, evidenced by $2-4$ times slower ${ }^{1} \mathrm{H}$ polarization transfer from water to macromolecules (Supplementary Fig. 6). The lignin and polysaccharide peaks in maize secondary cell walls only reached $20-30 \%$ of the equilibrium intensity within $4-\mathrm{ms}^{1} \mathrm{H}$ mixing (Supplementary Fig. 6). This water-buildup rate is notably slower than in the primary cell walls of Arabidopsis, which, at 4-ms, exhibits as high as $60-80 \%$ and $30-40 \%$ intensities for pectins and cellulose, respectively ${ }^{32}$. This can be attributed to the deposition of hydrophobic lignin, decreased water content ${ }^{8}$, tighter packing of 
a

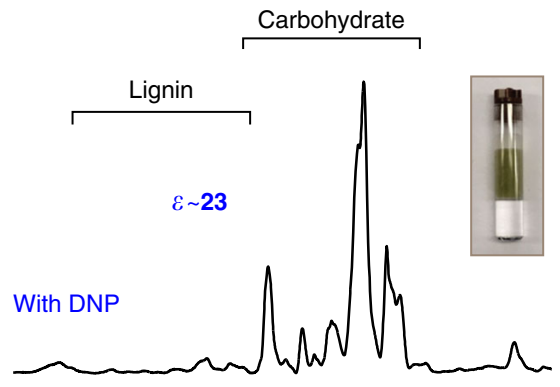

No DNP, $\times 2$

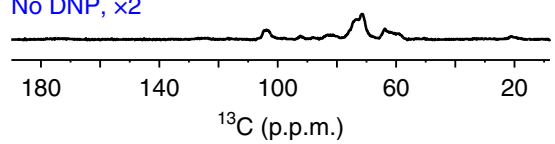

b

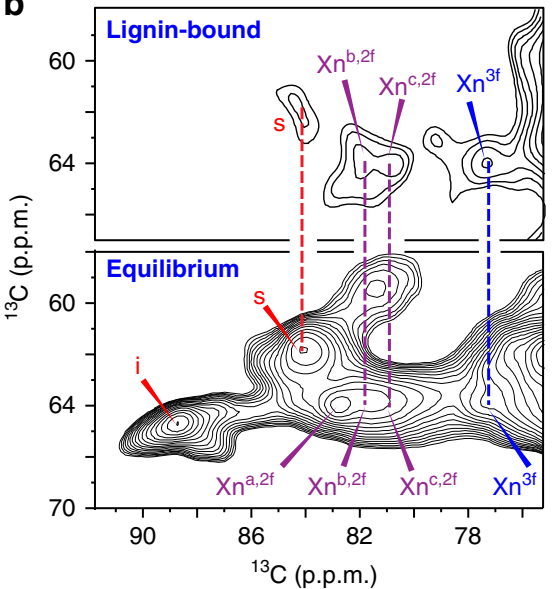

Fig. 4 DNP reveals the conformational selectivity of xylan for lignin-binding. a DNP enhances the NMR sensitivity by 23 -fold on maize. The inset shows a representative DNP sample. $\mathbf{b}$ Lignin-edited (top) and control (bottom) ${ }^{13} \mathrm{C}-{ }^{13} \mathrm{C}$ correlation spectra measured using DNP. The lignin-edited spectrum only shows polysaccharides spatially proximal to lignin, including three-fold and a subset of two-fold xylan (type b and c) and surface cellulose (s)
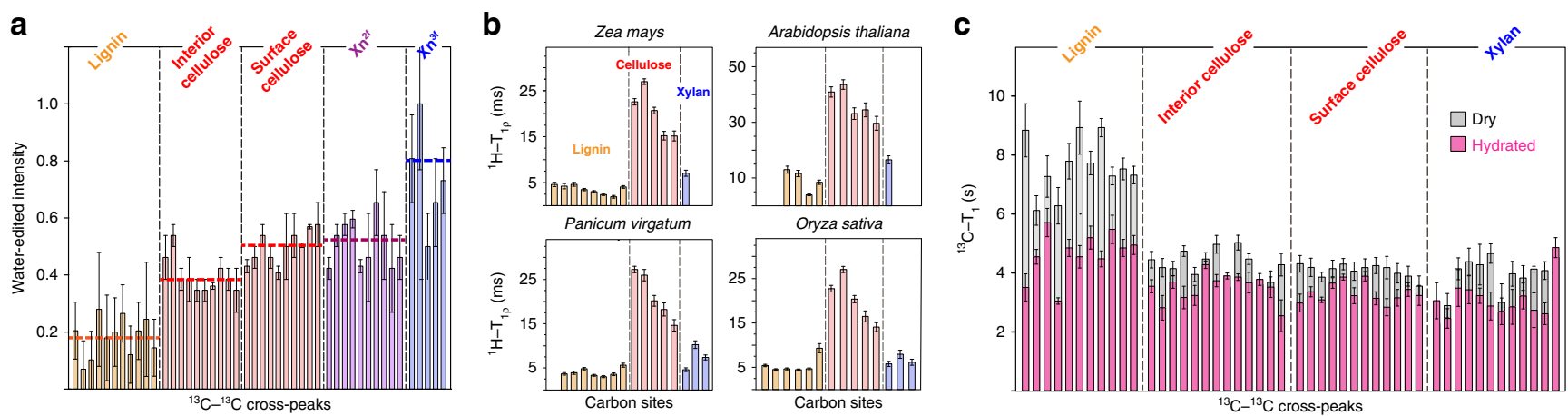

Fig. 5 Lignin is highly hydrophobic and dynamically distinct from polysaccharides. a Water-edited intensities showing the hydration level of molecules in maize. Error bars are standard deviations propagated from NMR sensitivity. The hydration level decreases in the order of three-fold xylan, 2-fold xylan, surface cellulose, interior cellulose and lignin. $\mathbf{b}{ }^{1} \mathrm{H}-\mathrm{T}_{1 \rho}$ relaxation times of four plants detect molecular motions on the microsecond timescale. ${ }^{13} \mathrm{C}_{-} \mathrm{T}_{1}$ relaxation times of hydrated (magenta) and dried (grey) maize reflect nanosecond timescale motions. Distinct from the polysaccharide, lignin has long ${ }^{13} \mathrm{C}$ $\mathrm{T}_{1}$ but short ${ }^{1} \mathrm{H}-\mathrm{T}_{1}$ relaxation. Error bars are standard deviations of the fit parameters. The $x$-axis corresponds to well-resolved cross peaks or carbon sites as tabulated in Supplementary Table 7-10. Source Data are provided as a Source Data file

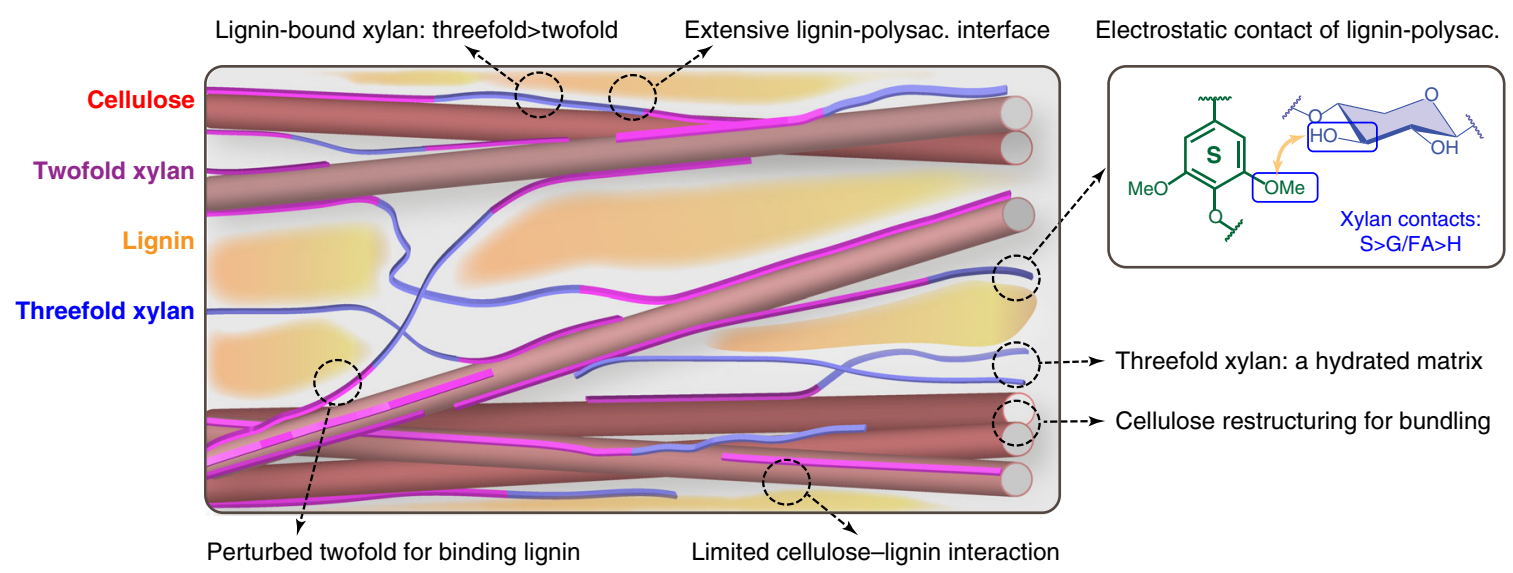

Fig. 6 A revised model of lignin-polysaccharide packing and secondary cell wall architecture. Cellulose microfibrils, two-fold and three-fold xylan, and lignin are depicted in red, purple, blue and yellow, respectively. The secondary cell wall adopts much tighter packing than the primary cell walls, and the two hydrophobic cores of lignin and cellulose are bridged by xylans in a conformation-dependent manner. The key findings of this study have been annotated in the model, with the corresponding spots highlighted using dashline circles. The representative polar motifs responsible for lignin-xylan interactions are boxed in blue. Polysaccharides are abbreviated as polysac. The depiction may not be to scale 
polymers, and lack of pectins with their water-stabilizing action. Tight packing also explains the unexpectedly large number of spatial contacts that are more prominent than those of highly porous primary cell walls ${ }^{33,34}$.

Lignin and polysaccharides are dynamically distinct. The domain separation of lignin and polysaccharide is confirmed by their distinct dynamics. Lignin has the shortest ${ }^{1} \mathrm{H}-\mathrm{T}_{1 \rho}$ relaxation times of all wall polymers (Fig. $5 \mathrm{~b}$ ) and its fast relaxation can be attributed to $\mu$ s motions similar to the ring flips of protein tyrosine or phenylalanine sidechains ${ }^{35}$ or the collective motions of multiple rings. Similar motions are not feasible in the hydrogenbonded glucan chains of cellulose microfibrils, which exhibit the longest ${ }^{1} \mathrm{H}-\mathrm{T}_{1 \rho}$ relaxation times. This trend is fully reversed for the ${ }^{13} \mathrm{C}-\mathrm{T}_{1}$ relaxation, with the longest times for lignin, $\sim 5 \mathrm{~s}$ for hydrated sample and $8 \mathrm{~s}$ for dried materials (Fig. 5c), indicating that aromatics resist rapid local reorientation on the ns timescale. In contrast, the ${ }^{13} \mathrm{C}-\mathrm{T}_{1}$ relaxation is markedly shorter for all polysaccharides due to efficient spin diffusion across the tightly packed polysaccharides. These dynamical heterogeneities support the concept of inhomogeneous mixing on the nm scale. The aromatic rings exhibit pronounced $\mu$ s motion, thus are unlikely to adopt the partial alignment observed in woods using FourierTransform Infrared (FTIR) and Raman spectroscopy ${ }^{36,37}$. In addition, lignin contains both rigid and mobile components (Supplementary Fig. 7, 8), indicative of the coexistence of aggregated rings and polysaccharide interfaces.

The fact that pronounced ${ }^{13} \mathrm{C}-\mathrm{T}_{1}$ differences between lignin and carbohydrates still exist in dehydrated samples suggests a large domain size far beyond the reach of relayed spin diffusion, a process that reduces relaxation heterogeneity for molecules within a few nanometers. Therefore, lignin domains may be as large as a few to tens of nanometers across so that the unique characteristics of motion and hydration can be fully retained.

\section{Discussion}

This study provides heretofore unavailable molecular evidence to define the 3D architecture of secondary cell walls. The exceptional resolution and sensitivity of ssNMR and DNP, assisted by a novel experiment for analyzing the lignin-carbohydrate interface, allow us to identify 234 intermolecular cross peaks that pinpoint the packing interactions, 325 relaxation curves that probe polymer mobilities on microsecond and millisecond timescales, and 62 site-specific hydration data (Supplementary Table 3-10). This large dataset markedly alters the paradigm for lignocellulose structure. The two hydrophobic cores of lignin and bundled cellulose are bridged by xylan in a conformation-dependent manner (Fig. 6): the dehydrated two-fold xylan with a flat-ribbon structure coats the microfibril surface ${ }^{15}$ and the remaining, wellhydrated xylans with uneven conformations connect the lignin nanodomains via extensive interfaces, along which electrostatic interactions occur between lignin methyl ethers and sugar polar groups. This well-defined boundary between aromatics and polysaccharides validates the modeling results in which lignin and xylans tend to self-aggregate to form separate phases, between which only limited interpenetration can happen ${ }^{30}$.

The numerous cross peaks between xylan and lignin, and between different carbon sites, mainly represent through-space, sub-nanometer contacts rather than covalent linkages. In fact, the lack of through-bond correlations between the arabinose sidechains of xylan and the ferulate lignin in the INADEQUATE spectrum (Fig. 1a, f) refines the hypothesis that xylan sidechains are crosslinked to lignin via ferulate or diferulate residues ${ }^{38,39}$. Such covalent bonding may only occur at limited spots, well below the detection limit of ssNMR. The abundant noncovalent interactions documented here predominate the physical interactions between xylan and lignin.

Pectin has also been assumed to be covalently connected to lignin via ester or ether linkages and bridge the lignin to hemicellulose $^{40}$. Pectin has been proposed to play a crucial role in lignification, especially in the initiation steps, as evidenced by biomimetic polymerization process of coniferyl alcohol in pectin solutions ${ }^{41-43}$. The intact stems analyzed here only contain a minor fraction of pectic substances and we have not identified any lignin-pectin cross peaks. Therefore, the proposed covalent linkages between lignin and pectin are scarce, if they exist, or only happening at the early stage of lignin deposition. These two molecules are not extensively co-localized within the subnanometer scale in the mature plant stems.

The structure that emerges from this ssNMR study differs substantially from contemporary views of complex lignocellulose in four aspects. First, lignin is found to bind mainly xylans rather than cellulose. For decades, lignin has been considered as the glue that connects cellulose microfibrils with hemicellulose ${ }^{44}$. The minor cellulose-lignin interaction observed here largely discounts previous models in which cellulose aggregates are proposed to be directly coated by lignin ${ }^{10}$ but rather suggests that lignin and cellulose are spaced and joined by xylan. Second, the phaseseparation of lignin and xylan has revised an earlier model, where these two polymers are depicted to be well-mixed via entanglements and covalent linkage ${ }^{11}$. Third, we have emphasized the importance of electrostatic interactions over the hydrophobic contact, which is another possible mechanism proposed by simulation $^{23}$. Finally, we have revealed, for the first time, that distorted xylan structure favors lignin-binding. This discovery, integrated with the previous finding that flat xylan conformers bind cellulose ${ }^{16}$, has fully revealed the structure-function relationship for xylan and resolved how this versatile hemicellulose can bridge different molecules with diverse conformational structures. These novel molecular characteristics provide the structural basis for designing more digestible crops and further optimizing the biomass degradation process to facilitate the production of biorenewable energy. Similar approaches can be applied to biomasses from other plants and organisms.

\section{Methods}

Isotope labeling and preparation of plant materials. The uniformly ${ }^{13} \mathrm{C}$-labeled stems $\left(97 \%{ }^{13} \mathrm{C}\right)$ of four plants were prepared by IsoLife (Wageningen, The Netherlands) using the following protocol. Briefly, uniformly ${ }^{13} \mathrm{C}$-enriched $(97$ atom $\%{ }^{13} \mathrm{C}$ ) plant stems of maize (Zea mays; Age 2 months after sowing), rice (Oryza sativa; Age 7 months), switchgrass (Panicum virgatum; Age 3.5 months), and Arabidopsis thaliana, (ecotype Columbia-O; Age 1 months) were produced under identical growth conditions in custom-designed, air-tight, high-irradiance labelling chambers of the Experimental Soil Plant Atmosphere System. Plants were grown hydroponically under controlled environmental conditions: photosynthetic photon flux density 900 (700 for A. thaliana) $\mu \mathrm{mol} \mathrm{m} \mathrm{m}^{-2} \mathrm{~s}^{-1}$ (top of plants), $16 \mathrm{~h}$ ( $A$ th. $14 \mathrm{~h}$ ) day length, day/night temperature $24 / 16^{\circ} \mathrm{C}$, $\mathrm{RH} 75 \%$, in a closed atmosphere containing 97 atom\% ${ }^{13} \mathrm{CO}_{2}\left(\mathrm{CO}_{2}\right.$ enriched with the stable isotope ${ }^{13} \mathrm{C}$; from pressurized cylinders, Cambridge Isotope Laboratories) from germination till harvest. Minerals and water were supplied as a modified Hoagland-type nutrient solution with micronutrients ${ }^{45}$ and iron as Fe(III)-EDTA ${ }^{46}$, modified to contain a maximum of $25 \%$ of total $\mathrm{N}$ as ammonium, maintaining $\mathrm{pH}$ between 5 (maize: 4) and 6. At harvest, immediately after removing the plants from the growth chamber, plant shoots were dissected into leaves and stems, cut to $2-4 \mathrm{~cm}$ pieces, weighed, packaged in food-grade $\mathrm{PE}$ pouches, and stored at $-30^{\circ} \mathrm{C}$. After freeze-drying, representative subsamples were prepared for ${ }^{13} \mathrm{C}$ analysis by high-abundance isotope ratio mass spectrometry (IRMS; Stable Isotope Facility, UC-Davis, CA, USA). ${ }^{13} \mathrm{C}$ abundance of $\mathrm{CO}_{2}$ in the chamber atmosphere was kept close to 97 atom $\%{ }^{13} \mathrm{C}$, continuously monitored during culture by non-dispersive infra-red detection. All the four plant samples were hydrated to $40 \mathrm{wt} \%$ and $80-100 \mathrm{mg}$ were sliced and packed into a $4 \mathrm{~mm}$ Bruker magic-angle spinning (MAS) NMR for ssNMR experiments. Around $30 \mathrm{mg}$ of maize were packed into a 3.2-mm Revolution NMR rotor. Another $28 \mathrm{mg}$ of maize was proceeded in DNP matrix and transferred to a 3.2-mm sapphire rotor for MAS DNP experiments as detailed below. 
ssNMR experiments for resonance assignment. Solid-state experiments were conducted on a Bruker Avance $600 \mathrm{MHz}(14.1$ Tesla) spectrometer and a $400 \mathrm{MHz}$ (9.4 Tesla) Bruker Avance spectrometer using 3.2-mm and 4-mm MAS HCN probes respectively. Most experiments except those with MAS-DNP were collected under $10-14 \mathrm{kHz}$ MAS at $294-298 \mathrm{~K} .{ }^{13} \mathrm{C}$ chemical shifts were externally referenced to adamantane $\mathrm{CH}_{2}$ signal at $38.48 \mathrm{ppm}$ on the TMS scale. Typical radiofrequency field strengths were $80-100 \mathrm{kHz}$ for ${ }^{1} \mathrm{H}$ decoupling and hard pulse, $62.5 \mathrm{kHz}$ for ${ }^{1} \mathrm{H} \mathrm{CP}$ and $50-62.5 \mathrm{kHz}$ for ${ }^{13} \mathrm{C}$.

To assign the NMR signals of polysaccharides and lignin, 2D double-quantum (DQ) correlation spectra were recorded using the refocused CP J-INADEQUATE pulse sequence ${ }^{28,47}$, which relies upon the scalar coupling between two ${ }^{13} \mathrm{C}$ nuclei to obtain through-bond information regarding directly couple ${ }^{13} \mathrm{C}$ nuclei. A $2 \mathrm{D}$ ${ }^{13} \mathrm{C}-{ }^{13} \mathrm{C}$ correlation spectrum is also measured with $53-\mathrm{ms}$ CORD mixing ${ }^{48}$. All the assigned ${ }^{13} \mathrm{C}$ chemical shifts of both polysaccharides and lignin have been summarized in Supplementary Table 2.

To analyze the lignin composition in four different plants, spectral deconvolution of the ${ }^{13} \mathrm{C}$ quantitative DP spectra was conducted using the software DMfit $^{49}$. The peak area of the $159 \mathrm{ppm}, 153 \mathrm{ppm}$ and $147 \mathrm{ppm}$ were assigned to the H4, S2/6 and the mixture of G3/4 and FA3/4/7, respectively (Supplementary Fig. 1c). To convert the peak area into molar percentage, the carbon numbers of each peak and the residue multiplicity need to be considered. We have thus divided the relative intensity of $\mathrm{S} 2 / 6$ by 2 , and the G3/4 and FA3/4/7 peak intensity by either 2 or 3 to account for the two extreme conditions. The resulting error margin was well below $3 \%$ for the $\mathrm{H}$ and $\mathrm{S}$ residues in all plants and below $5 \%$ for the G/FA residues.

The aromatic signals of lignin are typically difficult to analyze in solids due to the signal suppression caused by large chemical shift anisotropy. Here we employ a modified version of the standard proton-driven ${ }^{13} \mathrm{C}$ spin diffusion (PDSD) method that reintroduces ${ }^{13} \mathrm{C}-{ }^{1} \mathrm{H}$ dipolar coupling via a gated decoupling period to enhance lignin signals against the proton-rich polysaccharides (Supplementary Fig. 3,4$)^{50}$. A total dipolar dephasing period of $68 \mu$ s is employed to reintroduce ${ }^{13} \mathrm{C}-{ }^{1} \mathrm{H}$ dipolar couplings that selectively suppress the signals of protonated carbons. This dipolar dephasing period is asymmetric with respect to the $\pi$ pulse in the Hahn echo, containing two undecoupled delays of $46 \mu \mathrm{s}$ and $22 \mu \mathrm{s}^{51}$. A $100 \mathrm{~ms}$ mixing period is applied to both the gated-PDSD and the standard DARR experiment. The standard DARR experiment better detects the protonated carbons due to the use of CP while the gated-PDSD preferentially detects the nonprotonated carbons. Adding these two spectra with the same number of scans better presents the lignin signals due to the compensation of spectral asymmetry caused by the proton density heterogeneity in the aromatic motifs.

\section{ssNMR experiments for structural determination. To determine lignin-}

carbohydrate interactions, 2D gated PDSD experiments were measured on all four plant samples using $100 \mathrm{~ms}$ mixing time for detecting intramolecular cross peaks and $1 \mathrm{~s}$ for long-range intermolecular cross peaks. 74, 62, 59, and 39 intermolecular cross peaks have been identified for the rice, Arabidopsis, switchgrass, and rice, respectively. These 234 long-range cross peaks were categorized into 87 strong, 68 medium and 79 weak cross peaks according to the relative area of each single cross peak within a whole $1 \mathrm{D}{ }^{13} \mathrm{C}$ cross-section. For the 74 cross peaks in maize, they include 26 strong, 24 medium and 24 weak cross peaks. The intensity cutoff is set to $>5.0 \%$ for strong restraints, $2.5-5.0 \%$ for medium restraints, and $<2.5 \%$ for weak restraints (Supplementary Table 3-6). This strategy has been recently employed to determine the structure of fungal cell walls ${ }^{52}$. These structural restraints, together with the lignin-edited spectra measured using MAS-DNP (see below), provide site-specific information of the cell wall packing.

To determine the site-specific hydration of lignin and polysaccharides, we conducted the water-edited $2 \mathrm{D}{ }^{13} \mathrm{C}-{ }^{13} \mathrm{C}$ correlation experiment (Supplementary Fig. $4 \mathrm{c})^{32,53}$, which generated 62 hydration restraints. This experiment uses a ${ }^{1} \mathrm{H}$ $\mathrm{T}_{2}$ relaxation filter of $0.71 \mathrm{~ms} \times 2$ to suppress the polysaccharide signals to less than $2 \%$ but retains $75 \%$ of water magnetization. The water-polarization is further transferred to spatially proximal polysaccharides using a $2.25-\mathrm{ms}{ }^{1} \mathrm{H}$ mixing period and a $1 \mathrm{~ms}{ }^{1} \mathrm{H}-{ }^{13} \mathrm{C} \mathrm{CP}$ for ${ }^{13} \mathrm{C}$ detection. A $100-\mathrm{ms}$ DARR mixing period is used for both the water-edited spectrum and the control $2 \mathrm{D}{ }^{13} \mathrm{C}-{ }^{3} \mathrm{C}$ correlation spectrum showing full intensity. Both the control and water-edited $2 \mathrm{D}$ spectra are plotted with normalization to the worst hydrated iC4-iC6 cross peaks at $(89,65$ ppm). Both spectra are plotted using a bottom level that is set to $20 \%$ of the iC4iC6 peak height, a level increment for multiplication of 1.2 and 28 contour lines. The intensity ratio between the $2 \mathrm{D}$ water-edited spectrum and the control spectrum is quantified, which is further normalized by that of the $\mathrm{Xn}^{3 f_{2} / 3-4}$ cross peak with the highest water-edited intensity (Supplementary Table 7). The 2D spectra are processed using QSINE window function (SSB 2.5) for the polysaccharide region and Gaussian Multiplication (LB, GB: $-30,0.03$ ) for the lignin region. A series of $1 \mathrm{D}$ water-buildup curves are also measured using a ${ }^{1} \mathrm{H}-\mathrm{T}_{2}$ relaxation filter of $1 \mathrm{~ms} \times 2$ and a ${ }^{1} \mathrm{H}$ mixing period that varies from $0.1 \mu$ s to 100 $\mathrm{ms}$ to obtain the water-to-polysaccharide/lignin buildup curves.

To systematically determine the molecular mobility, we have generated 325 relaxation curves (Supplementary Table $8-10$ ) by measuring $1 \mathrm{D} / 2 \mathrm{D}{ }^{13} \mathrm{C}$ spinlattice $\left(T_{1}\right)$ relaxation and ${ }^{1} \mathrm{H}$ rotating-frame spin-lattice relaxation $\left(T_{1 \rho}\right)$ relaxation at $298 \mathrm{~K}$ under $10 \mathrm{kHz}$ MAS on the $400-\mathrm{MHz}$ spectrometer. The ${ }^{1} \mathrm{H}-\mathrm{T}_{1 \rho}$ is measured using a $62.5 \mathrm{kHz}$ for spin-lock. The ${ }^{13} \mathrm{C}-\mathrm{T}_{1}$ is measured using both the standard inversion recovery and the $\mathrm{z}$-filter versions ${ }^{54}$. The inversion recovery experiment is measured using a long recycle delay of $30 \mathrm{~s}$, thus providing quantitative detection of all molecules. The Torchia $\mathrm{T}_{1}$ measurement is CP-based, thus preferentially probes the rigid molecules. Furthermore, we have measured a series of $2 \mathrm{D}{ }^{13} \mathrm{C}-{ }^{13} \mathrm{C}$ correlation experiment with a variable $\mathrm{z}$-filter time to probe the ${ }^{13} \mathrm{C}-\mathrm{T}_{1}$ relaxation with enhanced resolution (Supplementary Fig. $4 \mathrm{~d}$ ) ${ }^{55}$. For the dried sample, 9 of $2 \mathrm{D}$ spectra were measured using $\mathrm{z}$-filter times of $0,0.1,0.4,1,2$, $3.5,5,7.5$ and $10.5 \mathrm{~s}$, and the total experimental time is $89 \mathrm{~h}$. For the hydrated sample, 7 spectra were measured using $\mathrm{z}$-filter times of $0,0.1,0.4,1,2,3.5$ and $5.5 \mathrm{~s}$, and the total experimental time is longer, $118 \mathrm{~h}$, due to an increase in the number of scans. The intensity of each cross peak in the 2D spectra was quantified and plotted as relaxation curves. These data were fit using a single exponential decay function to obtain the site-specific relaxation times.

The dynamical profile of cell wall components is also examined using a series of one-dimension (1D) ${ }^{13} \mathrm{C}$ spectra measured using four different methods on a $600 \mathrm{MHz}$ spectrometer under $14 \mathrm{kHz}$ MAS. Refocused INEPT ${ }^{56}$ were measured using a total polarization of $5.74 \mathrm{~ms}$, which consists of two delays of $1.72 \mathrm{~ms}$ followed by two delays of $1.15 \mathrm{~ms}$. $1 \mathrm{D}{ }^{13} \mathrm{C}$ direct polarization (DP) spectra were measured using a $2 \mathrm{~s}$ recycle delay for the detection of dynamic components and a $40 \mathrm{~s}$ recycle delay to obtain quantitative detection of cell wall molecules. The difference spectrum was obtained by subtracting the $2 \mathrm{~s}$ DP spectrum from the $40 \mathrm{~s}$ DP spectrum with normalization by the number of scans, and this difference spectrum only reports rigid molecules. $1 \mathrm{D}^{13} \mathrm{C}$ cross polarization $(\mathrm{CP})$ spectra were measured with 1 -ms contact time, which preferentially improves the sensitivity of the rigid molecules.

MAS-DNP sample preparation. The maize stem sample was processed for MAS DNP experiments ${ }^{57-59}$. The stock solution of AMUPol radical ${ }^{60}$, the DNP matrix, was prepared using a solvent mixture of $\mathrm{d}_{8}$-glycerol/ $\mathrm{D}_{2} \mathrm{O} / \mathrm{H}_{2} \mathrm{O}(60 / 30 / 10 \mathrm{Vol} \%)$ and a radical concentration of $10 \mathrm{mM}$. About $28 \mathrm{mg}$ of the ${ }^{13} \mathrm{C}$ maize sample was impregnated into $60 \mu \mathrm{L}$ of the AMUPol solution and grinded for 15-20 min to allow the radical to penetrate through the plant cell wall. $28 \mathrm{mg}$ of well-hydrated plant sample was then transferred into a 3.2-mm sapphire rotor. The NMR sensitivity has been enhanced by 23 -fold with and without microwave irradiation. The lignin has shorter DNP buildup time ( $2.1 \mathrm{~s}$ ) compared with polysaccharides $(3.7 \mathrm{~s})$ indicating a better association with the paramagnetic radicals. Three possible reasons might account for this preferential binding: (1) radicals may be better trapped in the aromatic network of lignins during the hand grinding of biomass in DNP matrix; (2) the polysaccharide cores are so tightly packed that the radicals, with the largest dimension of $\sim 13 \AA^{60}$, cannot effectively penetrate through; 3 ) the lignin-coating on the carbohydrate cores substantially decrease the surface-tovolume ratio of polysaccharide complex, which also explains the decrease in polysaccharide accessibility upon lignin deposition.

MAS-DNP experiments. The DNP experiments were carried out on a $600 \mathrm{MHz} /$ $395 \mathrm{GHz}$ MAS-DNP spectrometer using a $3.2 \mathrm{~mm}$ probe under $10 \mathrm{kHz}$ MAS frequency. The cathode currents of the gyrotron were $120 \mathrm{~mA}$ and the temperature was $104 \mathrm{~K}$ with microwave on. The gyrotron microwave source was equipped with a shutter to program the duration of microwave irradiation during the experiments $^{61}$

With the sensitivity enhancement from DNP, we are able to design a novel spectral editing experiment that combines dipolar and frequency filter to achieve clean selection of the aromatic signals of lignin over the signals (Supplementary Fig. 4b), the highest peak of which is 270 times higher than lignin peaks (Supplementary Fig. $3 \mathrm{~d}-\mathrm{g}$ ). The microwave is on during the recycle delay and the $\mathrm{CP}$ excitation but turned off by the shutter after $\mathrm{CP}$ to prevent repolarization of the dominant signals. A short recycle delay of $2.7 \mathrm{~s}$ is also applied to further enhance the lignin signals over the polysaccharides that have a longer DNP buildup time of $3.7 \mathrm{~s}$. The selected lignin polarization is transferred to polysaccharides using a 0.5 -s PDSD mixing period and the signals of these lignin-proximal polysaccharides are detected as a $2 \mathrm{D}{ }^{13} \mathrm{C}-{ }^{13} \mathrm{C}$ correlation spectrum with $20-\mathrm{ms}$ PDSD mixing. The carrier frequency was set to $153 \mathrm{ppm}$ for dipolar and frequency filters but changed to $114 \mathrm{ppm}$ during the $t_{1}$ evolution and $t_{2}$ detection. A control spectrum with 20 ms PDSD is also measured for comparison. The DNP measurement time is $41 \mathrm{~h}$ for the lignin-edited $2 \mathrm{D}$ and $2 \mathrm{~h}$ for the control spectrum.

Reporting Summary. Further information on experimental design is available in the Nature Research Reporting Summary linked to this article.

\section{Data availability}

All relevant data that support the findings of this study are available within the article and supplementary information or on reasonable request from the corresponding author. The Source Data underlying Figs. $2 \mathrm{~b}, 3 \mathrm{~b}-\mathrm{d}$, and $5 \mathrm{a}-\mathrm{c}$ and Supplementary Figs $2 \mathrm{a}-\mathrm{c}, 7$ and $8 \mathrm{f}$ are provided as a Source Data file.

Received: 11 October 2018 Accepted: 19 December 2018

Published online: 21 January 2019 


\section{References}

1. Somerville, C., Youngs, H., Taylor, C., Davis, S. C. \& Long, S. P. Feedstocks for Lignocellulosic Biofuels. Science 329, 790-792 (2010).

2. Service, R. F. Cellulosic ethanol-Biofuel researchers prepare to reap a new harvest. Science 315, 1488-1491 (2007).

3. Ragauskas, A. J. et al. The path forward for biofuels and biomaterials. Science 311, 484-489 (2006)

4. Poovaiah, C. R., Nageswara-Rao, M., Soneji, J. R., Baxter, H. L. \& Stewart, C. $\mathrm{N}$. Altered lignin biosynthesis using biotechnology to improve lignocellulosic biofuel feedstocks. Plant Biotechnol. J. 12, 1163-1173 (2014).

5. Vanholme, R., Morreel, K., Ralph, J. \& Boerjan, W. Lignin engineering. Curr. Opin. Plant Biol. 11, 278-285 (2008).

6. Loque, D., Scheller, H. V. \& Pauly, M. Engineering of plant cell walls for enhanced biofuel production. Curr. Opin. Plant Biol. 25, 151-161 (2015).

7. Fernandes, A. N. et al. Nanostructure of cellulose microfibrils in spruce wood. Proc. Natl Acad. Sci. USA 108, E1195-E1203 (2011).

8. Cosgrove, D. J. \& Jarvis, M. C. Comparative structure and biomechanics of plant primary and secondary cell walls. Front. Plant. Sci. 3, 204 (2012).

9. Salmen, L. Micromechanical understanding of the cell-wall structure. C. R. Biol. 327, 873-880 (2004)

10. Terashima, N., Yoshida, M., Hafren, J., Fukushima, K. \& Westermark, U. Proposed supramolecular structure of lignin in softwood tracheid compound middle lamella regions. Holzforschung 66, 907-915 (2012).

11. Langan, P. et al. Common processes drive the thermochemical pretreatment of lignocellulosic biomass. Green. Chem. 16, 63-68 (2014).

12. Lawoko, M. \& van Heiningen, A. R. P. Fractionation and Characterization of Completely Dissolved Ball Milled Hardwood. J. Wood Chem. Technol. 31, 183-203 (2011).

13. Cheng, K., Sorek, H., Zimmermann, H., Wemmer, D. E. \& Pauly, M. Solutionstate 2D NMR spectroscopy of plant cell walls enabled by a dimethylsulfoxided(6)/1-ethyl-3-methylimidazolium acetate solvent. Anal. Chem. 85 3213-3221 (2013).

14. Terashima, N. et al. 2D-NMR (HSQC) difference spectra between specifically ${ }^{13} \mathrm{C}$-enriched and unenriched protolignin of Ginkgo biloba obtained in the solution state of whole cell wall material. Holzforschung 63, 379-384 (2009).

15. Grantham, N. J. et al. An even pattern of xylan substitution is critical for interaction with cellulose in plant cell walls. Nat. Plants 3, 859-865 (2017)

16. Simmons, T. J. et al. Folding of xylan onto cellulose fibrils in plant cell walls revealed by solid-state NMR. Nat. Commun. 7, 13902 (2016).

17. Dupree, R. et al. Probing the molecular architecture of arabidopsis thaliana secondary cell walls using two- and three-dimensional ${ }^{13} \mathrm{C}$ solid state nuclear magnetic resonance spectroscopy. Biochemistry 54, 2335-2345 (2015).

18. Newman, R. H., Hill, S. J. \& Harris, P. J. Wide-angle $x$-ray scattering and solid-state nuclear magnetic resonance data combined to test models for cellulose microfibrils in mung bean cell walls. Plant Physiol. 163, 1558-1567 (2013).

19. Haigler, C. H. et al. Molecular Modeling and Imaging of Initial Stages of Cellulose Fibril Assembly: Evidence for a Disordered Intermediate Stage. PlOS ONE 9, e93981 (2014).

20. Li, S. D. et al. Cellulose synthase complexes act in a concerted fashion to synthesize highly aggregated cellulose in secondary cell walls of plants. Proc. Natl Acad. Sci. USA 113, 11348-11353 (2016).

21. Mansfield, S. D., Kim, H., Lu, F. C. \& Ralph, J. Whole plant cell wall characterization using solution-state 2D NMR. Nat. Protoc. 7, 1579-1589 (2012).

22. Komatsu, T. \& Kikuchi, J. Comprehensive Signal Assignment of ${ }^{13} \mathrm{C}$-Labeled Lignocellulose Using Multidimensional Solution NMR and ${ }^{13} \mathrm{C}$ Chemical Shift Comparison with Solid-State NMR. Anal. Chem. 85, 8857-8865 (2013).

23. Houtman, C. J. \& Atalla, R. H. Cellulose-Lignin Interactions (A Computational Study). Plant Physiol. 107, 977-984 (1995).

24. Musel, G. et al. Structure and distribution of lignin in primary and secondary cell walls of maize coleoptiles analyzed by chemical and immunological probes. Planta 201, 146-159 (1997).

25. Bonawitz, N. D. et al. Disruption of Mediator rescues the stunted growth of a lignin-deficient Arabidopsis mutant. Nature 509, 376-380 (2014).

26. Rossini, A. J. et al. Dynamic Nuclear Polarization Surface Enhanced NMR Spectroscopy. Acc. Chem. Res. 46, 1942-1951 (2013).

27. Ni, Q. Z. et al. High frequency dynamic nuclear polarization. Acc. Chem. Res. 46, 1933-1941 (2013).

28. Perras, F. A. et al. Atomic-level structure characterization of biomass pre- and post-lignin treatment by dynamic nuclear polarization-enhanced solid-state NMR. J. Phys. Chem. A 121, 623-630 (2017).

29. Can, T. V., Ni, Q. Z. \& Griffin, R. G. Mechanisms of dynamic nuclear polarization in insulating solids. J. Magn. Reson. 253, 23-35 (2015)

30. Charlier, L. \& Mazeau, K. Molecular modeling of the structural and dynamical properties of secondary plant cell walls: influence of lignin chemistry. J. Phys. Chem. B 116, 4163-4174 (2012).
31. Busse-Wicher, M. et al. The pattern of xylan acetylation suggests xylan may interact with cellulose microfibrils as a twofold helical screw in the secondary plant cell wall of Arabidopsis thaliana. Plant J. 79, 492-506 (2014).

32. White, P. B., Wang, T., Park, Y. B., Cosgrove, D. J. \& Hong, M. Waterpolysaccharide interactions in the primary cell wall of Arabidopsis thaliana from polarization transfer solid-state NMR. J. Am. Chem. Soc. 136, 10399-10409 (2014)

33. Wang, T., Park, Y. B., Cosgrove, D. J. \& Hong, M. Cellulose-pectin spatial contacts are inherent to never-dried arabidopsis thaliana primary cell walls: evidence from solid-state NMR. Plant Physiol. 168, 871-884 (2015).

34. Wang, T., Zabotina, O. \& Hong, M. Pectin-cellulose interactions in the Arabidopsis primary cell wall from two-dimensional magic-angle-spinning solid-state nuclear magnetic resonance. Biochemistry 51, 9846-9856 (2012).

35. Gall, C. M., Cross, T. A., Diverdi, J. A. \& Opella, S. J. Protein Dynamics by Solid-State NMR- Aromatic Rings of the Coat Protein in Fd Bacteriophage. Proc. Natl Acad. Sci. USA 79, 101-105 (1982).

36. Atalla, R. H. \& Agarwal, U. P. Raman Microprobe Evidence for Lignin Orientation in the Cell-Walls of Native Woody Tissue. Science 227, 636-638 (1985).

37. Olsson, A. M., Bjurhager, I., Gerber, L., Sundberg, B. \& Salmen, L. Ultrastructural organisation of cell wall polymers in normal and tension wood of aspen revealed by polarisation FTIR microspectroscopy. Planta 233, 1277-1286 (2011)

38. Grabber, J. H., Ralph, J. \& Hatfield, R. D. Model studies of ferulate-coniferyl alcohol cross-product formation in primary maize walls: implications for lignification in grasses. J. Agr. Food Chem. 50, 6008-6016 (2002).

39. Grabber, J. H., Ralph, J. \& Hatfield, R. D. Cross-linking of maize walls by ferulate dimerization and incorporation into lignin. J. Agr. Food Chem. 48, 6106-6113 (2000)

40. Jeffries, T. W. Biodegradation of lignin-carbohydrate complexes. Biodegradation 1, 163-176 (1990)

41. Donaldson, L. A. Lignification and lignin topochemistry-an ultrastructural view. Phytochemistry 57, 859-873 (2001).

42. Hao, Z. Y. \& Mohnen, D. A review of xylan and lignin biosynthesis: foundation for studying Arabidopsis irregular xylem mutants with pleiotropic phenotypes. Crit. Rev. Biochem. Mol. 49, 212-241 (2014).

43. Lairez, D. et al. Aggregation during coniferyl alcohol polymerization in pectin solution: A biomimetic approach of the first steps of lignification. Biomacromolecules 6, 763-774 (2005).

44. Hill, S. J., Franich, R. A., Callaghan, P. T. \& Newman, R. H. Nature's Nanocomposites: A New Look at Molecular Architecture in Wood Cell Walls. N. Z. J. For. Sci. 39, 251-257 (2009).

45. Smakman, G. \& Hofstra, R. J. J. Energy-Metabolism of Plantago-Lanceolata, as Affected by Change in Root Temperature. Physiol. Plant. 56, 33-37 (1982).

46. deVisser, R., Vianden, H. \& Schnyder, H. Kinetics and relative significance of remobilized and current $\mathrm{C}$ and $\mathrm{N}$ incorporation in leaf and root growth zones of Lolium perenne after defoliation: assessment by ${ }^{13} \mathrm{C}$ and ${ }^{15} \mathrm{~N}$ steady-state labelling. Plant Cell Environ. 20, 37-46 (1997).

47. Cadars, S. et al. The refocused INADEQUATE MAS NMR experiment in multiple spin-systems: Interpreting observed correlation peaks and optimising lineshapes. J. Magn. Reson. 188, 24-34 (2007)

48. Hou, G., Yan, S., Trebosc, J., Amoureux, J. P. \& Polenova, T. Broadband homonuclear correlation spectroscopy driven by combined R2(n)(v) sequences under fast magic angle spinning for NMR structural analysis of organic and biological solids. J. Magn. Reson. 232, 18-30 (2013).

49. Massiot, D. et al. Modelling one- and two-dimensional solid-state NMR spectra. Magn. Reson. Chem. 40, 70-76 (2002).

50. Schmidt-Rohr, K., Fritzsching, K. J., Liao, S. Y. \& Hong, M. Spectral editing of two-dimensional magic-angle-spinning solid-state NMR spectra for protein resonance assignment and structure determination. J. Biomol. NMR 54, 343-353 (2012).

51. Williams, J. K., Schmidt-Rohr, K. \& Hong, M. Aromatic spectral editing techniques for magic-angle-spinning solid-state NMR spectroscopy of uniformly ${ }^{13} \mathrm{C}$-labeled proteins. Solid. State Nucl. Magn. Reson. 72, 118-126 (2015).

52. Kang, X. et al. Molecular architecture of fungal cell walls revealed by solidstate NMR. Nat. Commun. 9, 2747 (2018).

53. Etzkorn, M. et al. Secondary structure, dynamics, and topology of a sevenhelix receptor in native membranes, studied by solid-state NMR spectroscopy. Angew. Chem. Int. Ed. Engl. 46, 459-462 (2007).

54. Torchia, D. A. Measurement of proton-enhanced ${ }^{13} \mathrm{C}-\mathrm{T}_{1}$ values by a method which suppresses artifacts. J. Magn. Reson. 30, 613-616 (1978).

55. Wang, T., Williams, J. K., Schmidt-Rohr, K. \& Hong, M. Relaxationcompensated difference spin diffusion NMR for detecting ${ }^{13} \mathrm{C}-{ }^{13} \mathrm{C}$ long-range correlations in proteins and polysaccharides. J. Biomol. NMR 61, 97-107 (2015). 
56. Elena, B., Lesage, A., Steuernagel, S., Bockmann, A. \& Emsley, L. Proton to carbon-13 INEPT in solid-state NMR spectroscopy. J. Am. Chem. Soc. 127, 17296-17302 (2005).

57. Lee, D., Hediger, S. \& De Paepe, G. Is solid-state NMR enhanced by dynamic nuclear polarization? Solid State Nucl. Magn. Reson. 66-67, 6-20 (2015).

58. Akbey, U., van Rossum, B. J. \& Oschkinat, H. Practical aspects of highsensitivity multidimensional C-13 MAS NMR spectroscopy of perdeuterated proteins. J. Magn. Reson. 217, 77-85 (2012).

59. Kirui, A. et al. Atomic resolution of cotton cellulose structure enabled by dynamic nuclear polarization solid-state NMR. Cellulose (2018). https://doi. org/10.1007/s10570-018-2095-6.

60. Sauvee, C. et al. Highly efficient, water-soluble polarizing agents for dynamic nuclear polarization at high frequency. Angew. Chem. Int. Ed. 52, 10858-10861 (2013).

61. Dubroca, T. et al. A quasi-optical and corrugated waveguide microwave transmission system for simultaneous dynamic nuclear polarization NMR on two separate 14.1 T spectrometers. J. Magn. Reson. 289, 35-44 (2018).

\section{Acknowledgements}

This work was supported by National Science Foundation through NSF OIA-1833040. The authors thank Drs. Riqiang Fu and Zhehong Gan for experimental assistance, Dr. Brittney Nagle for proving maize coleoptile walls, Dr. Hui Yang for providing the cellulose model and Dr. Carol Taylor for constructive suggestions. DJC, along with Drs. Nagle and Yang, were supported as part of the Center for Lignocellulose Structure and Formation, an Energy Frontier Research Center funded by the US Department of Energy, Office of Science, Basic Energy Sciences under award no. DE-SC0001090. The National High Magnetic Field Laboratory is supported by National Science Foundation through NSF/DMR-1644779 and the State of Florida. The MAS-DNP system at NHMFL is funded in part by NIH S10 OD018519 and NSF CHE-1229170.

\section{Author contribution}

X.K., A.K., F.M.V., and T.W. designed and conducted the NMR and MAS-DNP experiments. X.K. and A.K. prepared and optimized the DNP samples. X.K., A.K., D.J.C., and M.C.D.W. analyzed the experimental data. X.K., A.K., F.M.V., D.J.C., and T.W. wrote the manuscript.

\section{Additional information}

Supplementary Information accompanies this paper at https://doi.org/10.1038/s41467018-08252-0

Competing interests: The authors declare no competing interests.

Reprints and permission information is available online at http://npg.nature.com/ reprintsandpermissions/

Journal peer review information: Nature Communications thanks the anonymous reviewers for their contribution to the peer review of this work. Peer reviewer reports are available.

Publisher's note: Springer Nature remains neutral with regard to jurisdictional claims in published maps and institutional affiliations.

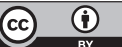

Open Access This article is licensed under a Creative Commons Attribution 4.0 International License, which permits use, sharing, adaptation, distribution and reproduction in any medium or format, as long as you give appropriate credit to the original author(s) and the source, provide a link to the Creative Commons license, and indicate if changes were made. The images or other third party material in this article are included in the article's Creative Commons license, unless indicated otherwise in a credit line to the material. If material is not included in the article's Creative Commons license and your intended use is not permitted by statutory regulation or exceeds the permitted use, you will need to obtain permission directly from the copyright holder. To view a copy of this license, visit http://creativecommons.org/ licenses/by/4.0/.

(c) The Author(s) 2019 